\title{
EL DERECHO AL AGUA LIMPIA \\ Y SANEAMIENTO Y SU INFLUENCIA EN \\ LA VIOLENCIA DE GÉNERO EN EL CAMPO \\ DE REFUGIADOS DE DADAAB (KENIA) ${ }^{1}$
}

\author{
AIDA FERNÁNDEZ GARCÍA-VALCÁRCEL ${ }^{2}$ \\ RAQUEL REGUEIRO DUBRA ${ }^{3}$
}

Fecha de recepción: junio de 2020

Fecha de aceptación y versión definitiva: septiembre de 2020

RESUMEN: El objetivo de esta investigación es analizar el derecho al agua limpia y saneamiento y su influencia en la violencia de género en el campo de refugiados de Dadaab (Kenia). Para ello se exploran los parámetros recogidos en la Observación General n. ${ }^{\circ} 15$ del Consejo Económico y Social de Naciones Unidas: disponibilidad, calidad y accesibilidad del agua y el saneamiento. Además, se estudian varias formas de violencia de género presentes en el campo que puedan estar relacionadas con el incumplimiento del Objetivo de Desarrollo Sostenible 6, relativo al agua limpia y saneamiento.

Palabras clave: Campo de refugiados de Dadaab; derecho al agua limpia y saneamiento; violencia de género; derechos humanos; Objetivos de Desarrollo Sostenible.

\section{The Right to Clean Water and Sanitation and its Influence on Gender-based Violence in the Dadaab Refugee Camp (Kenya)}

ABSTRACT: The aim of this paper is to analyse the right to clean water and sanitation and its influence on gender-based violence in the Dadaab refugee camp (Kenya).

\footnotetext{
1 Texto derivado de Trabajo de Fin de Grado.

2 Estudiante titulada del Grado en Relaciones Internacionales y del Grado en Traducción e Interpretación por la Universidad Pontificia Comillas. Correo electrónico: aida.fernan2@gmail.com.

3 Profesora del Departamento de Derecho Internacional Público de la Universidad Pontificia Comillas. Correo electrónico: rregueiro@icade.comillas.edu.
} 
To this end, the parameters set out in the United Nations Economic and Social Council's General Comment No. 15 are explored: availability, quality and accessibility of water and sanitation. In addition, this paper studies various forms of gender-based violence present in the camp that may be related to the failure to comply with Sustainable Development Goal 6 on clean water and sanitation.

KEY WORDS: Dadaab refugee camp; right to clean water and sanitation; gender-based violence; human rights; Sustainable Development Goals.

\section{INTRODUCCIÓN}

\subsection{El campo de Refugiados de DadaAb}

Desde el comienzo de la guerra civil en Somalia en 1991, el campo de refugiados de Dadaab se ha convertido en el hogar de miles de somalíes que buscan protección internacional en Kenia. En agosto de 2019 se contabilizaron 212936 refugiados y solicitantes de asilo en los campos de Dadaab, de los que aproximadamente la mitad son mujeres (ACNUR, 2019).

Al llegar a Dadaab, los refugiados somalíes se encuentran con serias dificultades. Entre ellas, una ausencia casi permanente de recursos naturales que dificulta las tareas diarias básicas, una inseguridad económica acuciante y ataques frecuentes de grupos armados o shifta (Horst, 2006, p. 57). Además, existen otros peligros como la violencia ejercida por el grupo paramilitar $\mathrm{Al}$ Shabab, una filial de Al Qaeda que opera en las inmediaciones del campo (p. 57). Dichas fuentes de conflicto han sido expuestas repetidamente ante organizaciones internacionales y gobiernos regionales y locales, y, como solución, se han impuesto mecanismos de protección de los derechos humanos, vigilados habitualmente por agencias de ayuda humanitaria como CARE o Médicos sin Fronteras (CARE, 2017).

Pese a los múltiples peligros que amenazan la vida en los campos de refugiados, el flujo migratorio de somalíes sigue activo en el Cuerno de África. El éxodo masivo desde Somalia en 2011 y la superpoblación de algunos asentamientos subrayan la importancia de los factores climáticos y la falta de agua como elementos clave en materia de migraciones (ACNUR, 2019). Las sequías llegan a Dadaab en forma de escasez de recursos y hambrunas, pero también agravan uno de los mayores problemas de la zona: el acceso al agua limpia y saneamiento, especialmente para las mujeres que, en una gran mayoría de sociedades, «tienen la responsabilidad principal del agua, el saneamiento y la salud en el hogar» (ONU-Agua, 2006, p. 1). 


\subsection{El DERECHO AL AgUA LIMPIA y SANEAMIENTO}

El derecho al agua limpia y saneamiento influye de manera inequívoca y transversal en la salud y el bienestar de las personas, no solo en cuestiones de higiene, consumo propio o alimentación, sino también en cuanto al disfrute de libertades económicas, sociales y culturales.

Aunque el derecho al agua limpia y al saneamiento se enmarca como tal en los Objetivos de Desarrollo Sostenible de Naciones Unidas para 2030 (Naciones Unidas, 2020), se encuentra de manera implícita en diversos textos normativos. Por un lado, está recogido en los artículos 3 (Todo individuo tiene derecho a la vida, a la libertad y a la seguridad de su persona) y 25 (Toda persona tiene derecho a un nivel de vida adecuado que le asegure, así como a su familia, la salud y el bienestar) de la Declaración Universal de los Derechos Humanos de 1948, aunque dicha Declaración no tenga valor vinculante per se, por lo que no crea, en el término más exacto de la palabra, derechos. Igualmente, el derecho al agua limpia y saneamiento se encuentra en el Pacto Internacional de Derechos Económicos, Sociales y Culturales (PIDESC) de 1966, cuyos artículos 11 y 12 versan, respectivamente, sobre el nivel de vida adecuado y la salud (De Luis Romero, Fernández Aller y Guzmán Acha, 2013, p. 220).

Además, encontramos menciones al derecho al agua limpia y saneamiento en tratados cuyo contenido es vinculante para los Estados que los ratifican, como la Convención para la Eliminación de Todas las Formas de Discriminación contra la Mujer (CEDAW) de 1979:

Artículo 14. 2. 2. Los Estados Partes adoptarán todas las medidas apropiadas para eliminar la discriminación contra la mujer en las zonas rurales a fin de asegurar, en condiciones de igualdad entre hombres y mujeres, su participación en el desarrollo rural y en sus beneficios, y en particular le asegurarán el derecho a: [...] h. Gozar de condiciones de vida adecuadas, particularmente en las esferas de la vivienda, los servicios sanitarios, la electricidad y el abastecimiento de agua, el transporte y las comunicaciones (Naciones Unidas, 1979, p. 7).

En ocasiones, la importancia del agua resta protagonismo al saneamiento, una condición básica vital para eliminar enfermedades y epidemias y proporcionar un acceso seguro a la higiene, especialmente en el caso de las mujeres. Según el catedrático Sánchez Bravo (2014), el saneamiento básico «permite eliminar higiénicamente las excretas y aguas residuales, y tener un medio ambiente limpio y sano tanto en la vivienda como en las proximidades de los usuarios» (p. 156). El acceso al saneamiento básico no solo se refiere a la tecnología que pueda llevar a tal fin, sino que también pretende dar seguridad y privacidad a sus usuarios. 


\subsection{LA VIOLENCIA DE GÉNERO Y LA VULNERACIÓN DE DERECHOS HUMANOS}

La violencia de género vulnera varios derechos humanos de la Declaración Universal de 1948 y los Pactos Internacionales de Derechos de 1996, tanto Civiles y Políticos como Económicos, Sociales y Culturales (Asamblea General de Naciones Unidas, 1966). Además de constituir una violación de los derechos más básicos de la Carta de 1948, como el derecho a la vida, a la libertad y a la seguridad de su persona (art.3) o el derecho a no ser sometida a torturas ni penas o a tratos crueles, inhumanos y degradantes (art.5), Nieves Rico, consultora de la Unidad Mujer y Desarrollo de la CEPAL (1996), considera que la violencia de género también vulnera el derecho de la igualdad ante la ley y el derecho a igual protección de la ley (art. 7), el derecho a recurrir a un tribunal imparcial (arts. 8 y 10) y el derecho a circular libremente (art. 13). Asimismo, Rico relaciona la violencia de género con la infracción del derecho a la libertad de opinión y de expresión (art. 19), debido a la anulación de la voz y autoridad de la mujer en dichas manifestaciones de violencia. También afecta a otros derechos de manera indirecta, como el derecho a la educación (art. 26) o el derecho de la libertad de reunión y asociación (art. 20), partes muy importantes del desarrollo personal y colectivo de las mujeres.

En la Conferencia Mundial de Derechos humanos de las Naciones Unidas en Viena (1993) se propone incluir en la Declaración Universal de los Derechos humanos una perspectiva de género y elaborar referencias específicas a la situación de las mujeres. Más recientemente, en 2010, la creación de ONU Mujeres permite desarrollar los temas de violencia de género en profundidad, y proporciona un espacio para buscar soluciones y ayudar al colectivo de forma directa. Respecto a la Agenda 2030, destacan el ODS 5 sobre la igualdad de género y el ODS 16 que, por primera vez, incluye «metas de erradicación de la violencia en todas sus formas y manifestaciones, con referencia expresa a la violencia sexual que sufren las mujeres en los conflictos armados, y a otras formas de violencia de género contra mujeres y niñas» (Mesa, 2014, p. 138).

En los asentamientos de Dadaab, la violencia de género es «especialmente común» (Izugbara, 2018, p. 4). Según el Comité Internacional de Rescate (2011, p. 109), el 43\% de las mujeres entre 15 y 49 años en Dadaab admitían haber sufrido algún tipo de violencia de género durante su vida, debido especialmente al conflicto, la sequía y las migraciones. 


\subsection{InTEgración de OBJetivos. Agua y GÉNero}

La igualdad entre hombres y mujeres respecto al acceso y disfrute del agua y del saneamiento ha sido uno de los objetivos de Naciones Unidas desde 1997 (ONU-Agua, 2006, p. 1). Las mujeres, responsables de la recolección de agua en aproximadamente el $80 \%$ de los hogares sin acceso a agua corriente (Naciones Unidas, 2020), suelen tener experiencia en temas como la calidad o el almacenamiento de agua. Sin embargo, a menudo las sociedades ignoran su papel central en la gestión de agua y recursos hídricos. Por este motivo, en las Conferencia de Mar del Plata en 1977, el Decenio Internacional del Agua Potable y el Saneamiento entre 1981 y 1990 y la Conferencia Internacional sobre el Agua y el Medio Ambiente (Declaración de Dublín, 1992) se reconoce expresamente el papel de las mujeres como proveedoras, custodias y gestoras del agua (Tortajada, 2007).

En los Objetivos de Desarrollo del Milenio ya se observa cierta relación entre el objetivo 7 ( (Garantizar la sostenibilidad del medio ambiente») y el objetivo 3 ( Promover la igualdad de género y el empoderamiento de la mujer»), en especial respecto al abastecimiento del agua para consumo doméstico y la gestión de recursos hídricos (Instituto Internacional del Agua de Estocolmo, 2005). En los Objetivos de Desarrollo Sostenible de la Agenda 2030 se observa una relación mucho más evidente entre agua y género: en la Agenda 2030 se considera que es importante mantener una perspectiva de género respecto a iniciativas que incluyan, especialmente, el saneamiento (ONU-Agua, 2006). Asimismo, en las metas del Objetivo 6 sobre agua limpia y saneamiento se intenta tener más en cuenta a las mujeres a la hora de crear infraestructuras: pequeños cambios en la ubicación y diseño de las letrinas, así como talleres de promoción de higiene en el colegio, podrían tener un gran impacto en la seguridad de mujeres y niñas en todo el mundo (p. 5). 


\section{FIGURA 1. INTEGRACIÓN DE LOS OBJETIVOS DE DESARROLLO SOSTENIBLE RELATIVOS A AGUA Y GÉNERO}

\begin{tabular}{|c|c|c|c|}
\hline \multicolumn{2}{|r|}{ Agua } & \multicolumn{2}{|r|}{ Género } \\
\hline $\begin{array}{l}\text { Objetivo } 6: \\
\text { agua limpia y } \\
\text { saneamiento }\end{array}$ & $\begin{array}{l}\text { Meta 6.1: De aquí a 2030, } \\
\text { lograr el acceso universal y } \\
\text { equitativo al agua potable } \\
\text { a un precio asequible para } \\
\text { todos } \\
\text { Meta 6.2: lograr el acceso a } \\
\text { servicios de saneamiento e } \\
\text { higiene adecuados y equi- } \\
\text { tativos para todos y poner } \\
\text { fin a la defecación al aire } \\
\text { libre, prestando especial } \\
\text { atención a las necesidades } \\
\text { de las mujeres y las niñas y } \\
\text { las personas en situaciones } \\
\text { de vulnerabilidad }\end{array}$ & $\begin{array}{l}\text { Objetivo 5: } \\
\text { Igualdad de } \\
\text { género }\end{array}$ & $\begin{array}{l}\text { Meta 5.1: Poner fin a todas } \\
\text { las formas de discrimina- } \\
\text { ción contra todas las mu- } \\
\text { jeres y las niñas en todo el } \\
\text { mundo } \\
\text { Meta 5.2: Eliminar todas las } \\
\text { formas de violencia contra } \\
\text { todas las mujeres y las ni- } \\
\text { ñas en los ámbitos público y } \\
\text { privado, incluidas la trata y } \\
\text { la explotación sexual y otros } \\
\text { tipos de explotación } \\
\text { Meta 5.a: Emprender refor- } \\
\text { mas que otorguen a las mu- }\end{array}$ \\
\hline $\begin{array}{l}\text { Objetivo 3: sa- } \\
\text { lud y bienestar }\end{array}$ & $\begin{array}{l}\text { Meta 3.9: reducir sustan- } \\
\text { cialmente el número de } \\
\text { muertes y enfermedades } \\
\text { producidas por productos } \\
\text { químicos peligrosos y la } \\
\text { contaminación del aire, el } \\
\text { agua y el suelo }\end{array}$ & & $\begin{array}{l}\text { a los recursos económicos, } \\
\text { así como acceso a la propie- } \\
\text { dad y al control de la tierra } \\
\text { y otros tipos de bienes, los } \\
\text { servicios financieros, la he- } \\
\text { rencia y los recursos natura- } \\
\text { les, de conformidad con las }\end{array}$ \\
\hline $\begin{array}{l}\text { Objetivo 11: } \\
\text { ciudades y } \\
\text { comunidades } \\
\text { sostenibles }\end{array}$ & $\begin{array}{l}\text { Meta 11.7: proporcionar } \\
\text { acceso universal a zonas } \\
\text { verdes y espacios públicos } \\
\text { seguros, inclusivos y acce- } \\
\text { sibles, en particular para } \\
\text { las mujeres y los niños, las } \\
\text { personas de edad y las per- } \\
\text { sonas con discapacidad }\end{array}$ & & $\begin{array}{l}\text { leyes nacionales } \\
\text { Meta 5.c: Aprobar y forta- } \\
\text { lecer políticas acertadas y } \\
\text { leyes aplicables para promo- } \\
\text { ver la igualdad de género y } \\
\text { el empoderamiento de todas } \\
\text { las mujeres y las niñas a to- } \\
\text { dos los niveles }\end{array}$ \\
\hline
\end{tabular}

Fuente: elaboración propia. Basada en los Objetivos de Desarrollo Sostenible para la Agenda 2030 (Naciones Unidas, 2020). Disponible en: https:/www.un.org/ sustainabledevelopment/es/objetivos-de-desarrollo-sostenible/

En los últimos años se ha promovido, a nivel internacional y local, la incursión de mujeres en iniciativas relacionadas con el agua y el saneamiento, ya que su aportación técnica y gestora en dichos proyectos resulta muy 
efectiva a la hora de establecer comunidades sostenibles (ONU-Agua, 2006, p. 6). De hecho, un estudio del Centro Internacional de Agua y Saneamiento (IRC, por sus siglas en inglés), demostró que, de los 88 proyectos llevados a cabo en 15 países, aquellos con participación de mujeres (tanto en diseño como en gestión) habían resultado más efectivos y sostenibles que aquellos sin participación femenina (Van Wijk-Sijbesma, 1998).

\section{5. ¿Cómo Se Relacionan agua y género en DadaAB?}

En el cuerno de África, la violencia causada por la escasez de agua está a la orden del día, debido en parte a las frecuentes sequías. Según un estudio del Programa de las Naciones Unidas para el Desarrollo (PNUD), uno de cada cuatro hogares somalíes había experimentado situaciones de violencia o conflicto tras la sequía de 2017 (Gobierno Federal de Somalia, 2017, p. 33). En ese mismo estudio se concluye que las mujeres y niñas, cuya actividad principal consiste en la búsqueda de recursos naturales, sufren de manera directa «responsabilidades desproporcionadas» durante los periodos de sequía (p. 34), tales como la necesidad de recorrer distancias más largas para encontrar agua, realizar trabajos no remunerados o convertirse en la fuente de ingresos principal para ayudar a su familia. En caso de inundaciones y desastres naturales, las mujeres también se ven más afectadas por desigualdades de género, debido a que, entre otras cosas, reciben menos información sobre los riesgos derivados de las catástrofes y se restringe su capacidad para evacuar zonas en riesgo por cargas sociales y culturales (ONU-Agua, 2006).

Respecto al saneamiento, las mujeres y niñas también se enfrentan a serias dificultades, ya que por un lado se ven obligadas a defecar o hacer sus necesidades al aire libre y tienen, por tanto, más posibilidades de sufrir ataques de violencia sexual (Thompson, Folifac y Gaskin, 2011, p. 111). Por otro lado, son más dependientes de los servicios de saneamiento para gestionar temas asociados con la menstruación y la maternidad, que ya implican de por sí una complicación debido a cargas culturales y religiosas (Sommer et al., 2014, p. 106).

Dentro de la violencia de género asociada al agua limpia y al saneamiento existen ejemplos de diversa índole, desde la violencia sexual hasta la violencia relativa al acceso al agua o la lucha por los recursos naturales. En Dadaab, el papel de las mujeres como proveedoras de recursos naturales puede dar pie incluso a una mayor probabilidad de violencia intrafamiliar, ya que se ha observado en diversos informes cómo la vulnerabilidad a la violencia al acceder a fuentes de agua crea un inevitable clima de «miedo, ansiedad y 
estrés» entre las mujeres y en las relaciones con sus parejas (Thompson et al., 2011, p. 115).

\section{6. ОвJeto del ESTUdio}

A la vista de los antecedentes expuestos, el presente estudio se centra en investigar el derecho al agua limpia y saneamiento y su influencia en la violencia de género en el campo de refugiados de Dadaab (Kenia). El trabajo se desarrolla en torno a dos cuestiones principales: la primera, el agua y el saneamiento, uno de los derechos básicos más importantes para una vida digna y saludable. La segunda, la protección de las mujeres, un grupo cuyos derechos humanos se pueden ver especialmente vulnerados en zonas de conflicto y reasentamiento como el campo de refugiados elegido.

El objetivo principal de esta investigación es el análisis de la situación del agua limpia y el saneamiento en Dadaab y su influencia de la violencia de género. Los objetivos específicos del estudio son:

- Averiguar cuál es el estado actual del agua limpia y el saneamiento en Dadaab de acuerdo con los parámetros establecidos por Naciones Unidas.

- Estudiar si existen diferencias entre los hombres y mujeres en cuanto al acceso al agua limpia y el saneamiento en el campo.

- Analizar diversas formas de violencia de género ya presentes en Dadaab.

- Determinar aquellas formas de violencia de género que puedan verse especialmente agravadas por la falta de agua limpia y saneamiento.

- Comprobar el cumplimiento de diversos derechos humanos en el campo relacionados con la protección de las mujeres.

Conviene destacar, no obstante, que en este trabajo no se busca establecer una relación directa entre los dos temas estudiados (agua y violencia de género), que resultaría imprecisa por la cantidad de variables existentes, sino que se trata más bien de exponer de manera detallada cómo la falta de agua o la negación del derecho al agua limpia y saneamiento podría actuar como agravante de varias formas de violencia de género ya presentes en el campo de refugiados de Dadaab (Kenia). 


\section{MÉTODO}

\subsection{INSTRUMENTOS}

Para llevar a cabo esta investigación se han analizado en profundidad diversos informes, tanto sobre la situación del agua y el saneamiento en Dadaab como sobre la violencia de género en el campo. Los informes proceden de organizaciones internacionales como ACNUR u organizaciones no gubernamentales como CARE, así como tesis doctorales y normativa legal. Se consultan los Objetivos de Desarrollo Sostenible, la Carta de Derechos Humanos de 1948 y otros documentos legales como la CEDAW, enmarcando la situación del campo en el marco legal internacional relativo a estándares de agua establecidos por la Observación n. ${ }^{\circ} 15$ del Consejo Económico y Social de Naciones Unidas.

A lo largo del trabajo se utilizan tanto herramientas de análisis de carácter cuantitativo, como herramientas de carácter cualitativo, que incluyen comentarios de personas refugiadas, descripciones derivadas de la observación directa, etc. La mayor parte de las fuentes analizadas son secundarias, debido a la limitación de extraer información de primera mano sobre la población refugiada. A lo largo del trabajo, se utilizan casos comparados de otras regiones del mundo, aunque se intenta acotar la investigación al campo de Dadaab, debido a la extensión limitada del estudio.

\subsection{Procedimiento}

En este trabajo se lleva a cabo una investigación analítica utilizando el método deductivo, ya que por un lado se revisan modelos teóricos como el marco legal del derecho al agua limpia y saneamiento y la violencia de género, y por otro se toman datos de fuentes primarias y secundarias para determinar la relación entre dichos documentos legales y la situación de mujeres refugiadas somalíes en el campo de refugiados de Dadaab.

Para comprobar si la situación actual se ajusta al Objetivo de Desarrollo 6 de Naciones Unidas, se utilizan los tres parámetros establecidos en la Observación General n. ${ }^{\circ} 15$ del Consejo Económico y Social de las Naciones Unidas para el agua limpia y el saneamiento. Dicha Observación estableció por primera vez categorías mínimas de disponibilidad, calidad y accesibilidad del agua y el saneamiento (2002, pp. 5-7). En función de esta clasificación se han propuesto tres parámetros: 
- Parámetro 1: Disponibilidad. Se presupone un abastecimiento de agua «continuo y suficiente» (2002, p. 5) para usos personales y domésticos como el consumo, la higiene personal y doméstica, el saneamiento o la colada. La cantidad disponible debería seguir las directrices establecidas por la Organización Mundial de la Salud (OMS) para cada caso.

- Parámetro 2: Calidad. Se presupone que el agua debe ser salubre y, por tanto, no debe contener microorganismos o sustancias químicas o radiactivas que amenacen la salud de las personas. El agua debe tener un color, un olor y un sabor aceptable para cada uso personal o doméstico.

- Parámetro 3: Accesibilidad. El agua debe ser accesible a todos, sin discriminación alguna.

- Accesibilidad física: el agua y las instalaciones y servicios de agua deben estar «al alcance físico de todos los sectores de la población» (p. 6). Todos los servicios deben ser de calidad y tener en cuenta las necesidades relativas al género, al ciclo vital y la intimidad. La seguridad física no debe verse amenazada durante el acceso a los servicios e instalaciones de agua. Para considerar que una persona tiene acceso a agua potable, la fuente debe estar a menos de 1 kilómetro de distancia del lugar de utilización, y la cantidad mínima obliga a recibir al menos «20 litros diarios para cada miembro de la familia» (Howard y Bertram, 2003).

- Accesibilidad económica: el agua debe ser asequible y los costes derivados del abastecimiento no deben comprometer ni poner en peligro el ejercicio de otros derechos.

Tras el análisis de cada parámetro, se incluye un apartado que versa sobre la influencia del agua limpia y el saneamiento en la violencia de género en Dadaab. Se analizan diversas manifestaciones de la violencia de género comprendidas en la CEDAW y dentro del marco la Carta de los Derechos humanos de Naciones Unidas de 1948 y de los Pactos Internacionales de Derechos Políticos, Civiles, Económicos, Sociales y Culturales. Se utilizan dichos artículos por su naturaleza de norma consuetudinaria y por haberse consolidado su aplicación en las últimas décadas como costumbre internacional. 


\section{RESULTADOS}

\subsection{Parámetro 1: Disponibilidad Del agua y del SANEAMiento}

En Dadaab se ha encontrado una falta de disponibilidad de agua. En 2009, el CDC (Centro para el Control y la Prevención de Enfermedades) denunció una «falta de capacidad técnica y coordinación» (p. 31) para garantizar la salud de los refugiados de Dadaab. El agua disponible no llegaba a los estándares internacionales de 20 litros diarios por persona, contrariamente a lo que aseguraban los informes de ACNUR (Chkam, 2016). Tampoco alcanzaba los estándares del proyecto Esfera, que se sitúan en 15 litros diarios por persona en campos de refugiados ${ }^{4}$ (2011). La sequía de 2011 agravó más todavía la situación, unida al hecho de que la población del campo no paraba de aumentar debido a la inseguridad en Somalia. No obstante, en una encuesta realizada por REACH en 286 hogares de Dadaab en 2018 (p. 6), un $88 \%$ de los encuestados consideraban haber recibido una cantidad adecuada de agua en los últimos 30 días, por lo que el sistema de reparto de agua puede haber cambiado entre 2009 y 2018.

De media, los hogares en Dadaab tienen acceso a 16,1 bidones por semana (CDC, 2009, p. 27). Se calcula que cada habitante utiliza 5,6 litros por semana, muy por debajo de los 20 litros diarios establecidos en los estándares internacionales (p. 27). Además, en cada hogar comparten agua, de media, 9,9 personas (p. 27). En la encuesta de CARE de 2009 se refleja también una falta de higiene, debido a la escasez de jabón y a la práctica de llevar los bidones de agua rodando por el suelo hasta las viviendas. Entre otras medidas de mejora se propone, por ejemplo, el uso de carretillas y una distribución más constante y eficiente de herramientas de aseo.

La falta de disponibilidad de agua puede influir en varias formas de violencia de género, entre las que destacan dos: la falta de independencia económica, social y cultural de la mujer y la violencia doméstica.

\subsubsection{Derecho 1: independencia económica, social y cultural}

El derecho a la independencia económica, social y cultural de las mujeres se ve reflejado en documentos legales de diversas maneras: aparece tanto en

4 El Proyecto Esfera se fundó en 1997 a manos de un grupo de organizaciones no gubernamentales y el Movimiento Internacional de la Cruz Roja y de la Media Luna Roja, con el objetivo de crear una regulación universal de cara a respuestas humanitarias: el Manual de Esfera. 
el artículo 20 de la Carta de Derechos Humanos de 1948 (Derecho a la libertad de reunión y asociación) como en el artículo 3 del Pacto Internacional de Derechos Económicos, Sociales y Culturales de 1966 (Los Estados Parte en el presente Pacto se comprometen a asegurar a los hombres y a las mujeres igual título a gozar de todos los derechos económicos, sociales y culturales enunciados en el presente Pacto).

Las mujeres de Dadaab, por su condición de recolectoras de agua, pueden ver reducido su tiempo de descanso o de trabajo productivo, lo que podría afectar a su independencia económica y social (Sánchez Bravo, 2014, p. 157). La dependencia social del hogar puede reducir el tiempo destinado a otras actividades comunitarias o a estrechar lazos con otras mujeres, dificultando así el derecho de la libertad de reunión y asociación en el campo. Por otro lado, la dependencia económica del hogar contribuye a perpetuar los roles de género establecidos por la sociedad somalí, que tradicionalmente restringen a las mujeres a los espacios privados del hogar y el cuidado de la familia (Population Council, 2009, pp. 10-12).

Además de restar independencia económica a las mujeres, el deber de recolectar agua puede implicar no asistir a la escuela. El derecho a la educación se encuentra tanto en el artículo 26 de la Carta de Derechos Humanos de 1948 como en el artículo 13 del Pacto Internacional de Derechos Económicos, Sociales y Culturales de 1966. Además, en el artículo 10 de la Convención sobre la Eliminación de Todas las Formas de Discriminación contra la Mujer (1979) se hace hincapié en la necesidad de los Estados de garantizar la igualdad de hombres y mujeres en cuanto al acceso, la orientación, las condiciones y las oportunidades de la esfera de la educación.

En Dadaab, algunas mujeres deben recorrer largas distancias para llegar a los pozos, y las colas convierten la tarea del agua en un gasto de tiempo y esfuerzo que puede disminuir el tiempo disponible para actividades educativas. En 2016, la tasa de escolarización de niñas en la educación secundaria en Dadaab era «alarmantemente baja» en comparación con la de los niños: las diferencias se reflejan con el Índice de Paridad de Género (IPG) en el acceso a la educación secundaria, cuyo nivel de paridad absoluta entre niños y niñas sería igual a 1, y en este caso apenas llegaba al 0,34, tal y como se indicaba en el Servicio de Información sobre Educación en Dadaab (Hassan Abdi, 2016, p. 47). La disponibilidad del agua también puede influir en la práctica de concertar matrimonios infantiles forzados: en un estudio llevado a cabo en Kenia (p. 47), uno de los participantes mencionó que se producían menos matrimonios infantiles cuando las familias tenían agua y comida suficiente, ya que «no veían la necesidad de casar a sus hijas para sobrellevar los problemas económicos». 
El acceso a la educación también influye en la percepción de la violencia de género entre las mujeres. La falta de acceso a la educación las hace más vulnerables frente a estigmas culturales y abusos: según un estudio realizado en Kenia, las mujeres que habían cursado únicamente la educación primaria eran más propensas a pensar que el comportamiento de las mujeres era la causa principal de la violencia de género, frente a aquellas con educación secundaria y superior, que identificaban con facilidad causas de violencia exógenas (Hassan Abdi, 2016, p. 41). Además, en otro estudio realizado a mujeres en Dadaab para analizar la percepción de la violencia de género, se encontró que el 91\% de las 186 encuestadas pensaban que la violación era la única manifestación de la violencia de género, y solo un 3\% identificaba la violencia doméstica como parte de la violencia de género (p. 41). De ellas, el 69\% de las mujeres no había recibido ningún tipo de educación, y solo el $15 \%$ habían llegado a la secundaria.

\subsubsection{Derecho 2: igualdad de género}

En el artículo 1 de la Carta de Derechos Humanos de 1948 se manifiesta la igualdad de todas las personas en dignidad y derechos, y en el artículo 23.4 del Pacto Internacional de Derechos Civiles y Políticos de 1966 se expresa el derecho a la igualdad de derechos y responsabilidades de ambos esposos en cuanto al matrimonio. Por otro lado, en el artículo 5 de la CEDAW se explicita que los Estados deberán combatir la desigualdad y los estereotipos en el hogar:

Los Estados Parte tomarán todas las medidas apropiadas para: a) Modificar los patrones socioculturales de conducta de hombres y mujeres, con miras a alcanzar la eliminación de los prejuicios y las prácticas consuetudinarias y de cualquier otra índole que estén basados en la idea de la inferioridad o superioridad de cualquiera de los sexos o en funciones estereotipadas de hombres y mujeres.

No obstante, la violencia doméstica en Dadaab, según Murray y Achieng (2011), «está extendida y, hasta cierto punto, aceptada en la comunidad» (p. 3). La falta de agua, al ser un recurso natural de primera necesidad, podría agravar los conflictos en el hogar. Hay estudios que indagan en cómo las tensiones por la escasez del agua repercuten en las relaciones de pareja: por ejemplo, en zonas rurales de Amhara, en Etiopía, se documentaron varias situaciones de violencia respecto al agua e incluso casos de violencia doméstica por la escasa cantidad de agua disponible para el hogar (Stevenson et al., 2012). Paralelamente, en una serie de estudios en zonas urbanas de Bolivia (Wutich y Ragsdale, 2008) se demostró que en los hogares con menos agua 
disponible las mujeres sufrían más la inseguridad derivada de esa escasez de agua. Entre otros efectos, la falta de agua se relacionaba directamente con problemas emocionales como enfado, molestia, miedo y preocupación entre las mujeres.

En dicho informe se sugería también que la inseguridad derivada de la falta de agua no estaba solo determinada por el acceso a las fuentes en sí, sino también por el estrés derivado de la negociación provocada por un reparto de agua desigual, responsabilidad que recaía especialmente sobre las mujeres. En otro estudio llevado a cabo entre comunidades dedicadas al pastoreo en Turkana, Kenia, se comprobó que las estrategias de supervivencia de las mujeres durante las sequías desencadenaban «una mayor exposición a abusos y actos violentos», tanto en el hogar como fuera (Chetail, Scarborough, Tesfaye y Gauntner, 2015). Según ACNUR (2008), la violencia doméstica no solo pone en riesgo la seguridad de las mujeres, sino que además limita su capacidad de moverse libremente, su acceso a servicios de ayuda y su contribución a actividades comunitarias con otras mujeres, entre ellas la recolección de agua.

\subsection{Parámetro 2: CALIDAD DEL AGUa y DEL SANEAMiEnto}

Con el objetivo de establecer los niveles de calidad del agua en Dadaab, el Plan de Seguridad de Aguas de CDC analizó en 2009 diversos estudios realizados acerca de las propiedades del agua. Primeramente, el Plan de Seguridad estudió el análisis completo que UNICEF Kenia pidió en 2004 a la organización GIBB Ltd sobre el acuífero de Merti, en Kenia, de cuyos 2,5 millones de metros cúbicos de agua se destina un « $40 \%$ a campos de refugiados, centros rurales y ganado» (p. 15). No obstante, una gran parte del acuífero presenta altos niveles de mineralización y salinización, y su agua no es, por tanto, apta para el consumo. Además, se recomendaba no bombear más del 60\% de su capacidad en menos de 10 horas (p. 15). Sin embargo, según los análisis de CDC, en 2009 (p. 16) existían 18 perforaciones en tres campos de Dadaab: en ellas se bombeaba un total de $4.851 \mathrm{~m}^{3} /$ día, cantidad que excedía las tasas de bombeo recomendadas en el estudio del acuífero y el uso permitido del agua, lo que repercutía tanto en la calidad del agua como en la degradación medioambiental de la zona.

Respecto a los indicadores de salubridad y potabilidad del agua, en 2009 se realizaban cinco pruebas por día para comprobar los niveles de cloro. Aunque el agua era potable, se encontraron múltiples problemas a lo largo de las pruebas, como «inconsistencias entre los trabajadores encargados de la 
tarea o fallos en la medición del cloro por desconocimiento» (p. 24). Además, los procesos de cloración no duraban tanto como deberían para garantizar la seguridad del agua: la OMS recomienda un $\mathrm{pH}$ inferior a 8 y una turbiedad inferior a 5 para una cloración eficaz, y en Dadaab los niveles de $\mathrm{pH}$ eran superiores a 8 (p. 24). Lo mismo sucedió con los niveles de plomo, que excedían tanto los niveles establecidos por la OMS $(0.01 \mathrm{mg} / \mathrm{L})$ como aquellos presentes en la regulación de Kenia $(0.05 \mathrm{mg} / \mathrm{L})$.

También se procedió a analizar el almacenamiento, el tratamiento y el trasvase del agua en las viviendas del campo. Respecto al almacenamiento, la situación en Dadaab era positiva: la mayoría de los hogares (un 80,4\% de los encuestados) almacenaban el agua durante un periodo inferior a un día: solo un $12,2 \%$ alegaba guardar el agua entre 1 y 2 días (p. 27). De la observación directa del equipo de análisis del Plan de Seguridad de Agua se extraía que el $81,3 \%$ de los bidones y contenedores observados en los hogares se consideraban en buen estado o limpios, aunque solo un 3\% de los encuestados alegaron tratar el agua de alguna forma antes de consumirla (p. 28).

La calidad de agua influye, sobre todo, en los riesgos sanitarios y la transmisión de enfermedades entre las mujeres de Dadaab. Procesos naturales como la maternidad requieren ciertos niveles de agua de calidad, y el uso de aguas deficientes puede tener efectos perjudiciales en la salud y el bienestar de los hogares.

\subsubsection{Derecho 3: disfrute de salud física y mental}

En el Pacto Internacional de Derechos Económicos, Sociales y Culturales de 1966 se reconoce «el derecho de toda persona al disfrute del más alto nivel posible de salud física y mental» (artículo 12). De la misma manera, en la Carta de Derechos Humanos de 1948 se recoge que «toda persona tiene derecho a un nivel de vida adecuado que le asegure, así como a su familia, la salud y el bienestar» (artículo 25.1.), considerando que «la maternidad y la infancia tienen derecho a cuidados y asistencia especiales» (artículo 25.2.). Además, la meta 3.9 del Objetivo de Desarrollo Sostenible 3 de Naciones Unidas busca «reducir sustancialmente el número de muertes y enfermedades producidas por productos químicos peligrosos y la contaminación del aire, el agua y el suelo» (2020).

La mala calidad del agua tiene una gran influencia en la salud de las personas, en particular respecto a la propagación de enfermedades como la diarrea, el cólera y otras enfermedades parasitarias (ONU-Agua, 2006). Los usos del agua influyen en la cantidad de agua de buena calidad disponible para el consumo y la higiene personal. En encuestas realizadas a habitantes 
de Dadaab sobre los usos del agua, los encuestados dieron la misma importancia a utilizar el agua para cocinar (93\%) que a usarla para la ablución y otros ritos religiosos (93\%), seguidos de cerca por el aseo (87\%), la colada $(80 \%)$ o el consumo directo $(60 \%)$. No obstante, existen diferencias en el uso de agua en los hogares: las mujeres adultas «usan y necesitan más agua que el resto de los miembros, ya que son las responsables principales de la gestión de los recursos de agua, higiene y saneamiento en los hogares» (WASH Sector Gender Focal Points, 2016, p. 17).

Las diferencias entre hombres y mujeres sugieren que dar la misma cantidad de agua por persona y día no siempre cubriría las necesidades de todos los miembros del hogar, puesto que las mujeres necesitan más agua que los hombres para asuntos de maternidad, menstruación e higiene personal. Las mujeres que no tienen otra opción utilizan en ocasiones el agua en mal estado para su propia higiene, lo que puede repercutir en «serios problemas de salud» (The Water Channel, 2020). En Dadaab, un 10\% de los hogares encuestados en 2009 reconoció haber cogido agua estancada «recogida de las inmediaciones del grifo para uso doméstico» (CDC, 2009, p. 27), si bien solo una de las familias declaró haberla utilizado para consumo directo.

\subsubsection{Derecho 4: la maternidad y la salud reproductiva}

Entre las formas de violencia de género más frecuentes en Dadaab no solo se citan la violencia doméstica o aquellos delitos de naturaleza sexual, sino que también se nombra la Mutilación Genital Femenina y la «violación del derecho de las mujeres de decidir acerca de su salud reproductiva» (Murray y Achieng, 2011, p. 3). La maternidad, por ejemplo, es una cuestión que puede influir en gran medida en el bienestar y la salud de las mujeres. Se trata de un proceso que requiere mayores cantidades de agua, higiene y saneamiento, y en el que es particularmente importante consumir agua de calidad. Por ejemplo, se han llevado a cabo estudios que encuentran asociaciones entre el consumo de aguas con exceso de cloro y el desarrollo de problemas durante la gestación, tales como abortos espontáneos o partos de bebés prematuros (Bove, Shim y Zeitz, 2002).

La maternidad es una cuestión relevante en Dadaab, ya que hay un gran número de mujeres embarazadas: de 286 hogares encuestados por REACH, en un 35\% residía al menos una mujer embarazada o lactante (REACH, 2018). Además, en caso de falta de agua, las mujeres embarazadas pueden tener menos agua disponible para su higiene personal, pues las estrategias prioritarias para hacer frente a la escasez de agua se reflejan de la siguiente manera: en un 79\% de los hogares «se reduciría el agua utilizada en prácticas 
de higiene», mientras que en un $71 \%$ «se buscaría agua en puntos de recolección más lejanos»(REACH, 2018, p. 2).

\subsection{Parámetro 3: accesibilidad del agua y El SANEAMiento}

En Dadaab, la accesibilidad física y económica al agua y al saneamiento no está garantizada. Respecto a la accesibilidad física, en la encuesta realizada por CDC para analizar el estado de la higiene y el saneamiento en Dadaab en 2009 , se encontró que el 95,1\% de los individuos entrevistados tenían problemas con respecto al suministro de agua en el campo, siendo las razones principales «una cola demasiado larga [...], un mal funcionamiento de los grifos o escasa presión del agua» (p. 27). En ese mismo estudio se encontró también que un hogar en Dadaab esperaba, de media, 3 horas y 12 minutos al día para conseguir agua en los grifos comunitarios (p. 28). Asimismo, varios encuestados enumeraron los siguientes problemas en relación con el suministro de agua: pocos grifos y bombas donde recolectar agua, corrupción de las instituciones encargadas del agua, largas distancias a los pozos o incluso rechazo vecinal e imposibilidad de conseguir agua por ser nuevos integrantes del campo (p. 28).

La mayor parte de los hogares encuestados por CDC (el 51,3\%) extraía agua del grifo comunal una vez al día, aunque «un número sustancial» del total de hogares, el 31,3\%, alegaba no coger agua ni siquiera en días alternos (2009, p. 28). También había hogares que recogían agua más de una vez al día (el 10\%). El reparto de agua también depende del asentamiento en cuestión y de la proximidad del hogar a los pozos del campo: por ejemplo, en la encuesta realizada por CDC en 2009 a hogares de Dadaab, la media obtenida del tiempo empleado desde el hogar hasta el pozo más cercano, a pie, era de 4 minutos. En otro estudio realizado en 2011 y 2012, el 77,6\% de los hogares se encontraban a menos de $1 \mathrm{~km}$ de la fuente de agua más cercana (Augustine, 2013, p. 40). En el estudio más reciente encontrado sobre el estado del agua en Dadaab, solo el 52\% de los hogares encuestados tardaba menos de 30 minutos en llegar al punto de recolección de agua (REACH, 2018).

Pese a los avances, todavía existen problemas que dificultan el acceso a fuentes y pozos salubres. Aunque el 97,6\% de los encuestados individualmente por CDC (2009, p. 26) declaró «obtener el agua del hogar de un grifo comunitario", en ocasiones los hogares adquieren cantidades extra de agua de fuentes no oficiales debido a la escasez. A nivel familiar, el 19,5\% de los hogares declaró comprar agua procedente de carros de burro, lo que, además de aumentar las probabilidades de mala calidad del agua, también pone de 
manifiesto una violación al principio de accesibilidad económica, puesto que no todas las familias tienen los recursos suficientes para comprar dichas cantidades (p. 26). Según un estudio de 2012, el ingreso per cápita diario en Dadaab es de 0,47 dólares: el 93,4\% de los hogares encuestados viven bajo el umbral de la pobreza, lo que influye decisivamente en su capacidad de acceder al agua y saneamiento no garantizado por agencias de ayuda humanitaria (Augustine, 2013, p. 54).

\subsubsection{Derecho 5: no sometimiento a torturas, penas o tratos crueles, inhumanos o degradantes}

Tanto en el artículo 5 de la Carta de Derechos Humanos de 1948 como en el artículo 7 del Pacto Internacional de Derechos Políticos y Civiles de 1966 se establece el derecho a no ser sometido a torturas ni a penas o tratos crueles, inhumanos o degradantes. Además, en estos dos documentos se recoge el derecho a la vida, a la libertad y a la seguridad (artículos 3 y 9, respectivamente). Por otro lado, en la CEDAW se insta a los Estados parte a «adoptar medidas adecuadas, legislativas y de otro carácter, con las sanciones correspondientes, que prohíban toda discriminación contra la mujer» (artículo 2b).

No obstante, tratos degradantes como la violencia sexual siguen siendo prácticas usuales en Dadaab, si bien pueden darse en diversas circunstancias. La violencia de género en Dadaab ha evolucionado durante las últimas décadas (Izugbara, 2018, pp. 4-6). Según este estudio, en 1998 los casos de violencia doméstica en Dadaab constituían más de la mitad del total de casos de violencia de género registrados por IRC y CARE (Crisp, 2000). Sin embargo, Izugbara (2018, p. 4) recalca que en los últimos años se ha percibido un aumento de violencia fuera del hogar, «en especial hacia mujeres solteras menores de 35 años y adolescentes, así como mujeres recién llegadas a los campos que no dispongan de una red social sólida».

Estos cambios en las formas de violencia implican que el entorno fuera del hogar tampoco tiene por qué ser seguro. En concreto, el parámetro de la accesibilidad del agua tiene importantes repercusiones en la seguridad de las mujeres. El principal problema es el acceso físico tanto a servicios de agua (colas, pozos, oficinas de gestión del agua) como de saneamiento (letrinas y baños).

En el acceso a servicios de agua son frecuentes los ataques a mujeres, entre ellos violaciones, agresiones sexuales y episodios de violencia. Por ejemplo, en el estudio de zonas rurales de Etiopía anteriormente mencionado (Stevenson et al., 2012), se hace hincapié en que las mujeres se exponen a más riesgos de violación al recoger agua de pozos lejanos. Igualmente, 
pueden sufrir episodios violentos en las colas mientras esperan, debido a la tensión provocada por la escasez de recursos. En Dadaab, además, en periodos de luchas entre clanes somalíes, los incidentes de violencia de género aumentan considerablemente, poniendo a las mujeres en el centro de los ataques, tal y como reflexiona Izugbara (2018, p. 8):

Acts of GBV were exacerbated by inter-clan conflicts in the camps [...]. In such situations, violence was perpetrated against women to humiliate their husbands, fathers, male relatives and their entire clan. [...] Women's bodies and sexuality are sites where men frequently assert power and dominance, expressed through rape, coerced sterilization, enforced nudity and other forms of genital violence, including mutilation.

El acceso al saneamiento es otro de los grandes retos en cualquier campo de refugiados. Diversos estudios en Dadaab concluyeron que las mujeres son «particularmente vulnerables a la violencia sexual cerca de las letrinas» (Sommer et al, 2014, p. 109). Los riesgos de violencia sexual en las inmediaciones de las letrinas aumentan por la falta de luz durante la noche y por la presencia de espacios abiertos para defecar (p. 109). En estudios publicados en 2013, las letrinas del campo de Dadaab eran aún mixtas, estaban poco alumbradas y no se podían cerrar con candado (Aboune y Hernandez, 2013, p. 23). Estas circunstancias ponían en peligro la seguridad de las mujeres, especialmente «a altas horas de la noche y al amanecer» (Murray y Achieng, 2011, p. 19).

En otro estudio realizado en 2012 (Augustine, 2013, p. 15), un 20\% de los 428 hogares encuestados en Dadaab reconocieron hacer sus necesidades entre arbustos por falta de letrinas disponibles. Para las mujeres, acudir a zonas abiertas es especialmente peligroso: además de estar socialmente restringidas a espacios privados, tener que ponerse en cuclillas las hace aún más vulnerables a ataques (Aboune y Hernandez, 2013, p. 110). Por otro lado, conviene recordar que la definición de accesibilidad según Naciones Unidas incluye, respecto a los usos del agua, que los servicios «deben tener en cuenta las necesidades relativas al género, al ciclo vital y la intimidad» (Consejo Económico y Social de Naciones Unidas, 2002). No obstante, la necesidad de privacidad durante la menstruación y la maternidad puede no verse satisfecha en el uso de los servicios de saneamiento, ya sea por la inseguridad asociada a las zonas de las letrinas o a su mal funcionamiento o escasez.

La capacidad de uso de las letrinas en Dadaab en 2011 y 2012, según las encuestas, no superaba el $50 \%$, ya que muchos servicios de saneamiento estaban bloqueados, olían mal o estaban llenos de moscas (Augustine, 2013, p. 45). El 58\% de los encuestados respondió, por otra parte, que la limpieza de las letrinas, al igual que aquella relacionada con la mayor parte de hogares 
y zonas comunitarias de los campos, seguía siendo una tarea de mujeres: el resto de encuestados no supo identificar quién se ocupaba de la limpieza (p. 45). Los avances de las organizaciones de ayuda humanitaria se reflejan en encuestas de 2018 (REACH, p. 2), cuando el 92\% de los hogares afirmaron tener acceso a letrinas para todos los miembros, aunque en dicha encuesta no se precisa el estado de las letrinas ni sus condiciones de seguridad. Respecto a la higiene, en 2012 se observó que un 30\% de los encuestados no se lavaban las manos correctamente tras ir al baño, y que un 10\% no se lavaba las manos nunca (Augustine, 2013, p. 7).

\subsubsection{Derecho 6: libertad de expresión e igualdad ante la ley}

La igualdad ante la ley, sin discriminación por motivos de raza, color, sexo, idioma, religión, opiniones políticas o de cualquier índole, origen nacional o social, posición económica, nacimiento o cualquier otra condición social aparece tanto en el artículo 7 de la Carta de Derechos Humanos de 1948 como en el Artículo 26 del Pacto Internacional de Derechos Políticos y Civiles de 1966. En estos dos documentos se encuentra también el derecho a la libertad de expresión y de opinión (artículos 19 y 19.2, respectivamente), que muchas veces influyen en el posterior acceso a servicios legales de protección.

De ciertos episodios de violencia física y sexual en el acceso al agua y al saneamiento se derivan otras formas de violencia de género: entre ellas, la falta de libertad de expresión para comunicar los hechos sucedidos y la desigualdad ante la ley a la hora de ser juzgadas. Aunque se ha hecho un gran esfuerzo por aumentar los mecanismos de denuncia de violencia de género en Dadaab, aún resulta muy difícil contabilizar los casos de violación y agresión sexual, ya que muchos no son denunciados por el estigma en la cultura somalí y por la presencia de sistemas informales como el Maslaha, que anulan a la mujer en cuanto a representación y acceso a los sistemas de justicia establecidos (Abdi, 2005).

Además, la escasa confianza en las instituciones y fuerzas de seguridad tampoco contribuye a crear un entorno seguro para denunciar: se han registrado casos de violaciones cometidas por la policía keniata estacionada en Dadaab para vigilar las inmediaciones (Abdi, 2005, p. 10). Esto plantea una «doble opresión» (p. 10) para los refugiados, y en especial para las mujeres: a la situación de crimen e inseguridad del campo se le suma la desconfianza en los sistemas de vigilancia. Pese a los esfuerzos realizados por parte del gobierno de Kenia respecto a la ratificación de la CEDAW y a sus propias medidas nacionales, en Dadaab sigue habiendo muchos casos de violencia 
de género que nunca llegan a denunciarse, debido a factores como barreras lingüísticas y aspectos culturales tales como la falta de conciencia sobre temas de género, que disminuyen la eficacia de los mecanismos de denuncia (Augustine, 2013).

En junio de 2015 se realizó una encuesta a 20 refugiados de Dadaab que se habían prestado a ser Trabajadores Comunitarios (Refugee Community Workers). Dichos trabajadores colaboran con las organizaciones encargadas de los servicios de ayuda a víctimas de violencia de género, que en el campo son IRC y CARE, y sirven de nexo entre estas y la población refugiada. Su participación logra que "más mujeres y niñas busquen y obtengan ayuda» en los servicios de atención a víctimas de violencia de género (Murray y Achieng, 2011). Entre otras actividades, los trabajadores comunitarios acompañan a las víctimas a denunciar, hacen servicios de interpretación y traducción y divulgan información sobre los mecanismos de ayuda para víctimas de violencia de género, por lo que conocen desde dentro la situación de las mujeres somalíes (Izugbara, 2018).

La percepción general de los trabajadores encuestados en 2015 indicaba que los incidentes de violencia de género en Dadaab habían disminuido en los últimos años. Entre los motivos de este descenso, se encontraban una mayor seguridad en los campos, más educación y conocimiento sobre la violencia de género, una menor llegada de nuevos migrantes o la disminución en conflictos entre clanes (p. 8). Los trabajadores aseguraban que los servicios de asistencia a víctimas de violencia de género son «cada vez más accesibles» en los campos, lo que permite aumentar los cuidados y la vigilancia de las víctimas. No obstante, y, pese a los avances, las encuestas sugerían que la mayor parte de los casos de violencia de género en Dadaab «ni se denuncian ni se documentan» (p. 8).

\section{DISCUSIÓN}

En este trabajo se ha investigado el derecho al agua limpia y saneamiento, enmarcado en el Objetivo de Desarrollo Sostenible n. 6 de Naciones Unidas, y se ha analizado su influencia en la violencia de género en el campo de refugiados de Dadaab (Kenia). La hipótesis propuesta supone que la falta de agua limpia y saneamiento puede agravar algunas formas de violencia de género presentes en Dadaab y, por tanto, influir de manera desproporcionada en la vida de las mujeres. 
Para ello se ha explorado, por una parte, el marco legal internacional y nacional donde se recogen tanto el derecho al agua limpia y al saneamiento como los derechos vulnerados por la violencia de género, utilizando para ello varios documentos legales y los Objetivos de Desarrollo Sostenible promulgados por Naciones Unidas. Por otra parte, se ha analizado la situación del agua y el saneamiento en Dadaab y se han estudiado aquellas formas de violencia de género que pueden verse agravadas por dicha falta de agua y saneamiento.

A lo largo de la investigación se ha comprobado que efectivamente existe cierta influencia de la falta de agua limpia y saneamiento en la violencia de género en el campo de refugiados de Dadaab, y que el incumplimiento del Objetivo de Desarrollo Sostenible 6 puede tener consecuencias respecto a diversas manifestaciones de violencia contra la mujer. Respecto a los tres parámetros establecidos por la Observación General n. ${ }^{\circ} 15$ de Naciones Unidas, se ha encontrado que el parámetro 1 (Disponibilidad del agua) podría tener cierta influencia en la independencia económica, social y cultural de las mujeres, así como en la igualdad de género. El parámetro 2 (Calidad del agua) está íntimamente relacionado con la salud física y mental, especialmente con la salud reproductiva, mientras que el parámetro 3 (Accesibilidad del agua y del saneamiento) puede tener cierta relación con el sometimiento a torturas, penas o tratos crueles, inhumanos o degradantes en el acceso a las instalaciones y respecto a la vulneración del derecho de libertad de expresión y de la igualdad ante la ley.

Respecto a la relación entre el acceso al agua y la violencia de género anteriormente mencionada, hay una acertada explicación en el informe de la Unión Internacional para la Conservación de la Naturaleza (UICN) sobre violencia de género (GBV, por sus siglas en inglés) y medioambiente (Castañeda Carney et al., 2020, p. 47):

It is important to note that a lack of water access is not a direct cause of GBV, but rather an amplifier of existing patterns. Having access to improved water facilities can reduce the risk of facing GBV during water collection, but not prevent other types of violence in other situations, let alone eradicate GBV.

En cuanto a las limitaciones de este trabajo, es imprescindible recordar que cuando se habla de Dadaab se está generalizando, ya que cada asentamiento particular (Ifo, Ifo II, Hagadera, y Dagahaley) tiene unas características propias respecto a la seguridad, las cantidades de agua disponibles o los niveles de violencia, de las que muchas veces no se tiene constancia. Además, en Kenia son los gobiernos de los condados los encargados de proveer a los ciudadanos con servicios de agua y saneamiento, y no el gobierno central. 
Los datos e informes sobre las prestaciones sanitarias se obtienen también de los condados para luego elaborar un informe nacional, lo que puede suponer un desequilibrio entre zonas urbanas y no urbanas. Por ejemplo, según el informe IMPACT n. ${ }^{\circ} 10$ para el periodo 2016/2017, elaborado por WASREB (Equipo de Regulación de Servicios de Agua), los servicios públicos de agua a nivel autonómico solo incluían al 48\% de la población de Kenia (21,8 millones); se había excluido a la población de zonas urbanas de bajos ingresos y zonas rurales no reguladas (IRC, 2018, p. 1). Este tipo de cuestiones políticas afecta a los datos obtenidos sobre el agua y el saneamiento y puede ser una limitación para contabilizar la disponibilidad del agua en el condado de Garissa, y especialmente en el campo de Dadaab.

Se debe mencionar que ha sido muy difícil encontrar información precisa y de calidad, tanto sobre la situación actual del agua y el saneamiento como acerca de la violencia de género en el campo de Dadaab. Esto puede deberse a varios motivos. En primer lugar, es difícil encontrar datos que muestren la evolución de un determinado problema a lo largo de los años. Por ejemplo, entre 2009 a 2018, dos años en los que se realizaron múltiples encuestas y trabajos de campo en Dadaab, hubo un cambio en las necesidades de la población y, por tanto, en los retos de las organizaciones presentes en el campo. En segundo lugar, los datos existentes de los informes de organizaciones internacionales y organizaciones no gubernamentales no siempre concuerdan. A lo largo del trabajo se ha observado en repetidas ocasiones que no siempre hay una respuesta unificada a los problemas del campo, y por lo tanto no hay un único análisis del cual extraer cifras y aspectos a mejorar.

Respecto al número total de casos de violencia de género relacionados con el acceso al agua limpia y saneamiento ha sido imposible conseguir datos, principalmente porque no hay apenas informes sobre el tema, y si los hay, no son de dominio público. Del intercambio de emails con Ruth Dede (2020), supervisora del departamento de Violencia de Género en Dadaab con DRC (Danish Refugee Council) se han extraído las siguientes conclusiones:

1. No es frecuente encontrar datos que integren de manera exhaustiva el derecho al agua limpia y al saneamiento y su influencia en la violencia de género en Dadaab. Es un tema poco estudiado debido a la complejidad que supondría su análisis y a la gran cantidad de variables que influyen en dicho estudio, tales como las circunstancias socioculturales de las poblaciones o la falta de profesionalidad de los técnicos al medir el impacto (Sommer et al., 2015).

2. En los últimos años se han tomado medidas para asegurarse de que el agua y el saneamiento sean accesibles para todos. No obstante, pese a las mejoras, no siempre se cumplen los estándares internacionales establecidos 
por Naciones Unidas y la Organización Mundial de la Salud para los campos de refugiados. Además, en esta afirmación solo se contempla el acceso, uno de los tres parámetros del marco internacional para medir el derecho al agua limpia y saneamiento, y no se hace referencia a la calidad del agua o a su disponibilidad, aunque se podría discutir el concepto de «acceso» como un genérico para referirse a la totalidad de los parámetros.

3. El abastecimiento de agua limpia y el saneamiento no son prioridades respecto a la protección de las mujeres en el campo. Es evidente que en el campo hay otros motivos de inseguridad más importantes para las mujeres, como los grupos armados o la inseguridad económica, pero debido a la falta de estudios sobre el tema no está claro hasta qué punto dicha inseguridad se pueda ver agravada, en ocasiones, por la vulneración del derecho al agua limpia y saneamiento.

Para futuras investigaciones, sería muy interesante estudiar otros factores de desigualdad de género relacionados con causas medioambientales, como la escasa presencia de mujeres en actividades agrícolas cuyo eje central es el agua, o las dificultades para heredar tierras de cultivo según ciertos sistemas legales. También resultaría de gran interés ampliar este trabajo con otras formas de violencia de género no estudiadas o trasladar este análisis a otros campos de refugiados en otras partes del mundo, contemplando siempre las diversas circunstancias económicas, sociales y culturales propias de cada región.

En conclusión, no solo es necesario eliminar toda discriminación hacia las mujeres, sino también fomentar su empoderamiento como agentes de cambio en la sociedad. Se insta especialmente a los gobiernos a contemplar las necesidades particulares del colectivo respecto a la intimidad, la higiene y el saneamiento, y a reforzar los mecanismos existentes para garantizar la seguridad y la protección de las mujeres y las niñas refugiadas en los campos de Dadaab y en los asentamientos temporales en todo el mundo.

\section{REFERENCIAS}

Abdi, M. (2005). En el limbo: dependencia, inseguridad e identidad entre los refugiados somalíes en los campamentos de Dadaab. Refugio: Canada's Journal on Refugees, 22(2), 6-14. Obtenido de https://refuge.journals.yorku.ca/index.php/refuge/ article/view/21328

Aboune, A., y Hernandez, J. (2013). Assessing refugee camp characteristics and the occurrence of sexual violence: A preliminary analysis of the Dadaab complex. Refugee Survey Quarterly, 32(4), 22-40. 
ACNUDH (2019). Naciones Unidas Consejo de Derechos Humanos. Recuperado el 23 de noviembre de 2019, del $39^{\circ}$ período de sesiones del Consejo de Derechos Humanos: https://www.ohchr.org/SP/HRBodies/HRC/RegularSessions/Session39/ Pages/ResDecStat.aspx

ACNUR (2008). Strengthening Refugee Protection, Assistance and Support to Host Communities in Kenya. ACNUR Kenia.

ACNUR (2019). ACNUR Kenia. Recuperado el 16 de octubre de 2019, de Dadaab Refugee Complex: https://www.unhcr.org/ke/dadaab-refugee-complex

ACNUR (Abril de 2016). ACNUR Comité español. Recuperado el 25 de 02 de 2020, de Declaración Universal de los Derechos Humanos: lista de artículos: https:// eacnur.org/blog/declaracion-universal-los-derechos-humanos-lista-articulos-tc_ alt45664n_o_pstn_o_pst/

Asamblea General de Naciones Unidas (20 de diciembre de 1993). Declaración sobre la eliminación de la violencia contra la mujer. Recuperado el noviembre de 6 de 2019, de https://www.ohchr.org/sp/professionalinterest/pages/violenceagainstwomen.aspx

Asamblea General de Naciones Unidas. (1966). Pacto Internacional de Derechos Civiles y Políticos. Naciones Unidas.

Asamblea General de Naciones Unidas. (1966). Pacto Internacional de Derechos Económicos, Sociales y Culturales. Naciones Unidas.

Augustine, A. (2013). Dadaab Water, Sanitation and Hygiene (WASH) Emergency Response End Term Evaluation Report 2011-2012. Nairobi: World Vision Kenya.

Bove, F. S. (2002). Drinking water contaminants and adverse pregnancy outcomes: a review. Environmental Health Perspectives, 110.

CARE (2017). CARE: Nuestras historias. Recuperado el 23 de octubre de 2019, de The Weight of Water in Kenya's Dadaab Refugee Camp: https:/www.care.org/impact/ stories/weight-water-kenyas-dadaab-refugee-camp

Castañeda Carney, I. L. (2020). Gender-based violence and environment linkages : the violence of inequality. Gland, Switzerland: UICN. doi:https://doi.org/10.2305/ IUCN.CH.2020.03.en

CDC (2009). Water Safety Plan for Dadaab Refugee Camps. CDC.

Chetail, S. S. (2015). Wealth \& Warriors: Adolescents in the Face of Drought in Turkana, Kenya. Oregon: Mercy Corps.

Chkam, H. (2016). Aid and the Perpetuation of Refugee Camps: The Case of Dadaab in Kenya 1991-2011. Refugee Survey Quarterly, 35(2), págs. 79-97. doi:doi:10.1093/ rsq/hdw005

Consejo Económico y Social de Naciones Unidas (2002). Observación General n. ${ }^{\circ} 15$ : El derecho al agua (artículos 11 y 12 del Pacto Internacional). Ginebra. Obtenido de https://agua.org.mx/wp-content/uploads/2017/06/Observacion-15_derecho_al_ agua.pdf

CPHD. (2011). The Forgotten Front: Water Security and the Crisis in Sanitation: Water in Informal Settlements. Kabul: Kabul University.

Crisp, J. (2000). 'A State of Insecurity: The Political Economy of Violence in Kenya's Refugee Camps'. African Affairs 99, 601-632. 
De Luis Romero, E. F., Fernández Aller y Guzmán Acha (2013). Derecho humano al agua y al saneamiento: derechos estrechamente vinculados al derecho a la vida. Documentación Social, 170.

Gobierno Federal de Somalia (2017). Somalia Drought Impact and Needs Assessment. Obtenido de https:/www.undp. org/content/dam/undp/library/Climate\%20 and\%20Disaster\%20Resilience/GSURR_Somalia\%20DINA\%20Report_Volume\%20I_180116_Lowres.pdf

Hassan Abdi, M. (2016). Assessment of Sexual and Gender Based Violence Reporting Procedures Among Refugees In Camps In Dadaab, Kenya (Research Project). Masters Of Arts In Rural Sociology And Community Development, University Of Nairobi (tesis no publicada).

Horst, C. (2006). Transnational Nomads: How Somalis Cope With Refugee Life in the Dadaab Camps of Kenya. New York: Berghahn Books.

Howard, G., y Bertram, J. (2003). Domestic Water Quantity,. Ginebra: Organización Mundial de la Salud.

Instituto Internacional del Agua de Estocolmo (2005). Millennium Project Task Force on Water and Sanitation, Health, Dignity and Development: What Will it Take? Estocolmo.

International Rescue Committee, (IRC). (2011). Gender-based Violence Rapid Assessment, Dadaab, Kenya. New York: IRC.

IRC (2018). National accountability mechanisms for SDG 6 in Kenya: a Briefing Paper. Obtenido de https://www.ircwash.org/sites/default/files/watershed_kenya_accountability_briefing_note.pdf

Izugbara, C. (2018). «Dicen que nuestro trabajo no es halal»: experiencias y desafíos de la comunidad de refugiados trabajadores involucrados en la prevención y atención de la violencia de género en Dadaab, Kenia. Revista de estudios sobre refugiados. Obtenido de http://eprints.lse.ac.uk/101626/1/They_Say_Our_Work_ Is_Not_Halal.pdf

Mesa, M. (2014). Retos inaplazables en el sistema internacional. Centro de Educación e Investigación para la Paz.

Murray, S., y Achieng, A. (2011). Gender-based Violence Assessment, Hagadera Refugee Camp at Dadaab, Kenya. Nueva York: IRC.

Naciones Unidas. (2020). Agenda 2030 - Objetivos de Desarrollo Sostenible. Recuperado el 5 de noviembre de 2019, de Gobierno de España: https://www.agenda2030. gob.es/es/objetivos

Naciones Unidas. (1979). Convención sobre la eliminación de todas las formas de discriminación contra la mujer. Obtenido de ACNUDH: https://www.ohchr.org/Documents/ProfessionalInterest/cedaw_SP. pdf

Naciones Unidas. (1948). Declaración Universal de Derechos Humanos. Naciones Unidas. Obtenido de https://www.ohchr.org/EN/UDHR/Documents/UDHR_Translations/spn.pdf

ONU-Agua (2006). Gender, Water and Sanitation: a Policy Brief. Recuperado el 2 de November de 2019, de https://www.un.org/waterforlifedecade/pdf/un_water_policy_brief_2_gender.pdf 
PNUD (2000). Objetivos de Desarrollo del Milenio. Obtenido de Programa de las Naciones Unidas para el Desarrollo: https://www.undp. org/content/undp/es/home/ sdgoverview/mdg_goals.html

Population Council (2009). An Assessment of Sexual and Gender-based Violence in Wajir District in North-Eastern Kenya. Reproductive Health Programme 1-22. Recuperado el 21 de noviembre de 2019

Prisca Dede, R. (2019). Questions about water and sanitation in Dadaab [correo electrónico].

REACH (2018). Multi-Sector Needs Assessment: Hagadera Refugee Camp. Garissa County.

Rico, N. (1996). Violencia de género: un problema de derechos. Serie Mujer y Desarrollo. 8-20.

Sánchez Bravo, Á. (2014). Injusticia Ambiental y Derecho Humano al Agua. Revista Thesis Juris, 155-164. doi:10.5585/rtj.v3i2.186.

Sommer, M., Ferron, S., Cavill, S., y House, S. (2014). Violence, Gender and WASH: spurring action on a complex, under-documented and sensitive topic. Environment and Urbanization, 27, págs. 105-116. Obtenido de https://journals.sagepub. com/doi/pdf/10.1177/0956247814564528

Stevenson, E. G., Greene, L. E., Maes, K. C., Ambelu, A., Tesfaye, Y. A., Rheingans, R., y Hadley, C. (2012). Water insecurity in 3 dimensions: an anthropological perspective on water and women's psychosocial distress in Ethiopia. Social Science and Medicine (1982), 75(2), 392-400. https://doi.org/10.1016/j.socscimed.2012.03.022

The Water Channel (2020). Why Gender Matters in IWRM. Recuperado el 16 de enero de 2020, de http://www.thewaterchannel.tv/gender/content/chapter5/5_1.html

Thompson, J. A., Folifac, F., y Gaskin, S. (2011). Fetching Water in the Unholy Hours of the Night: The Impacts of a Water Crisis on Girls' Sexual Health in Semi-urban Cameroon. Girlhood Studies. 4, 111-129.

Tortajada, C. (2007). El agua y el medio ambiente en las conferencias mundiales de las Naciones Unidas. Zaragoza: Centro de Documentación del Agua y el Medioambiente.

Van Wijk-Sijbesma, C. (1998). Gender in Water Resources Management, Water Supply and Sanitation: Roles and Realities Revisited. Delft, the Netherlands: International Research Centre for Water and Sanitation.

WASH Sector Gender Focal Points (2016). WASH Sector Gender Analysis Report in Za'atari and Azraq Refugee Camp. Naciones Unidas.

Wutich, A., y Ragsdale, K. (2008). Water insecurity and emotional distress: coping with supply, access, and seasonal variability of water in a Bolivian squatter settlement. Social science \& medicine (1982), 67(12), 2116-2125. Obtenido de https:// doi.org/10.1016/j.socscimed.2008.09.042 